\title{
Capacitación digital de los profesionales de museos en plena transformación digital
}

\section{Conxa Rodàa}

@innova2

\begin{abstract}
Resumen
Existe una gran necesidad de una mejor formación y perfeccionamiento en las capacidades digitales para que los museos puedan abordar con éxito los desafios y la transformación digital en el contexto actual. Lo digital debe abordarse de manera integral y estratégica dentro de las organizaciones. Cualquier rol debe estar preparado para lidiar con lo digital con suficiencia, por lo que el aprendizaje a lo largo de la vida será una constante.
\end{abstract}

Palabras clave: transformación digital, perfiles profesionales, formación, habilidades digitales.

\footnotetext{
Abstract

There is a great need for better training and refinement of digital skills so that museums can successfully address the challenges and digital transformation in today's context. Digital must be approached comprehensively and strategically within organizations. Any role must be prepared to deal with digital adequately, so lifelong learning will be a constant.
}

Keywords: digital transformation, professional profiles, training, digital skills. 


\section{Introducción}

Los museos son en general pobres en cuanto a recursos -económicos, tecnológicos, humanos. Pero los museos albergan dos grandes tesoros: las colecciones (su razón de ser) y la gente (lo que les llena de sentido). Con gente me refiero tanto al público, la audiencia, como al equipo interno, los profesionales del museo. Y son estos los que harán posible la transición digital. Al equipo del museo hay que valorarlo, hay que cuidarlo, cultivarlo y hay que darle herramientas.

Lo digital es un activador de la transformación, pero el cambio auténtico no ocurre solo debido a la tecnología. Requiere sobre todo una actitud abierta para ser parte activa del cambio necesario hoy para:

- evolucionar nuestros museos hacia una mejor actuación orientada a los públicos

- ser relevantes para la sociedad

- ser más una plataforma social que un proveedor unidireccional de conocimiento.

Como punto de partida veamos qué significa la transformación digital. La transformación digital es mucho más que digitalización. Transformación digital significa un enfoque integral y unificado que vincula la misión del museo con las necesidades y expectativas del público, potenciado por lo digital. Transformación digital consiste en aplicar la tecnología digital a una nueva manera de crear / producir / comunicar contenido y servicios. Afecta a toda la organización: a lo que hacemos y a cómo lo hacemos, a los procesos.

La auténtica revolución de los museos consiste en transformar la relación con los públicos, la interacción y la participación, la experiencia que se ofrece. Una transformación que se ve facilitada y reforzada por la vía digital.

Habitualmente me gusta definir el museo como conector, https://blog.museunacional.cat/es/museo-conector-publicos-contenidos) conector entre el conocimiento, los contenidos, la colección y los públicos. Con el público como eje. Alrededor de ese eje central hay múltiples derivadas: cómo se expone y se explica la colección, qué servicios y actividades ofrece el museo, cómo se gestiona internamente, cómo se invita a la comunidad a participar, todo para servir mejor a la misión del museo y a su audiencia. La transformación digital es más un cambio social que tecnológico. Lo digital hoy penetra en todos los rincones de actividad del museo y en cada contenido orientado al público: webs, colección online, móvil, redes sociales, museografía interactiva, comunicación, marketing, estrategia de contenidos, realidad inmersiva, etc. 


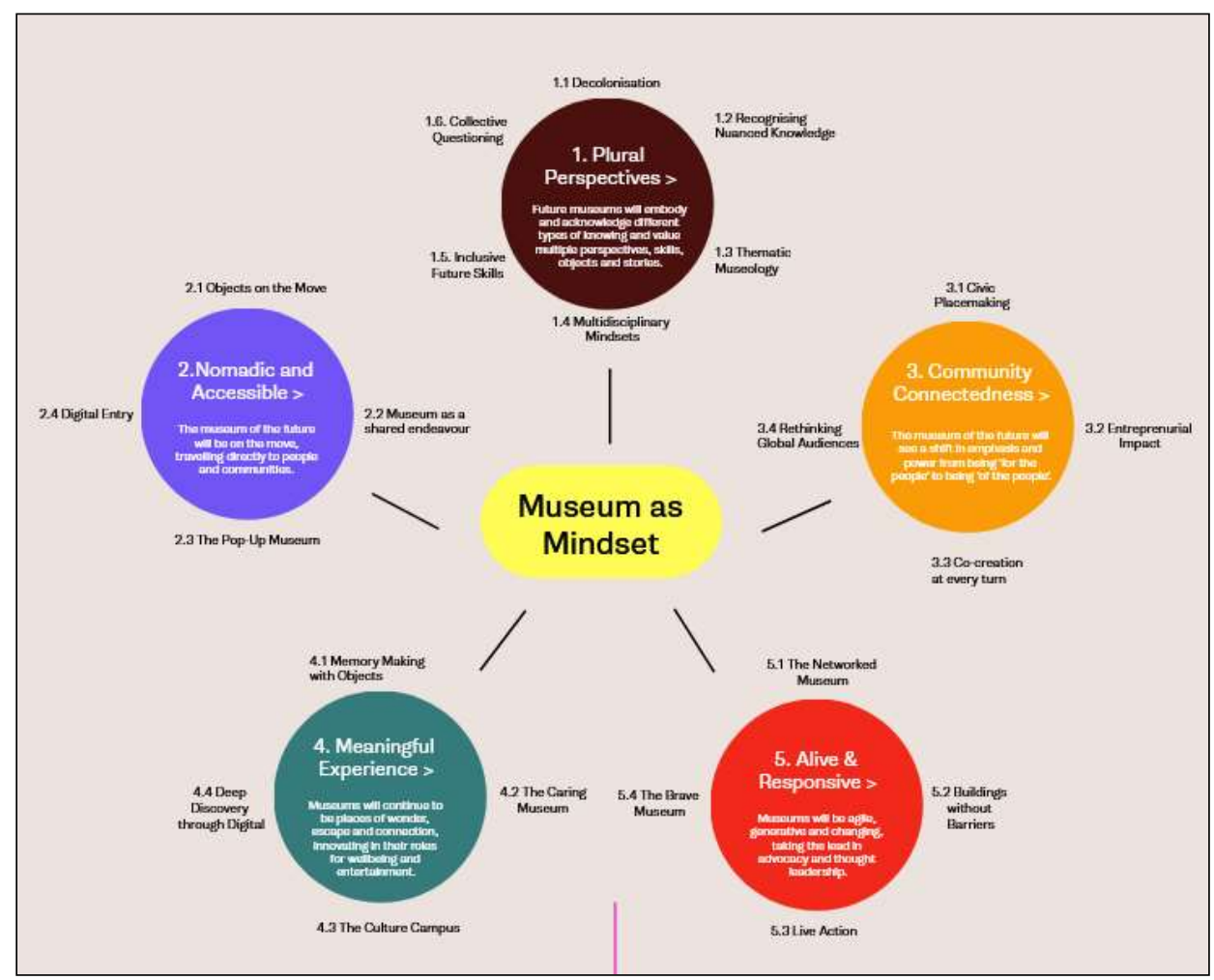

Fuente: museumofthefuture.com

Fig. 1 The Liminal Space (2020). "Mindsets for Museums of the Future"

Este gráfico me parece muy ilustrativo del momento de cambio que estamos viviendo. Me gusta especialmente la idea de museo como mindset, como "mentalidad". Eso comprende adoptar una perspectiva plural, ser accesibles, trabajar por la conexión con la comunidad, ofrecer experiencias con sentido, significativas, y, en definitiva, ser un museo vivo, inclusivo, responsivo a la sociedad. Lo digital contribuye a desarrollar y a fortalecer cada una de estas vertientes. Por ello se habla de la necesidad de impregnar de digital mindset, de mentalidad digital, a todo el museo.

Esto a nivel organizativo sacude muchos cimientos. El museo es un ecosistema donde todo está interconectado, todo está interrelacionado. Como la dimensión digital es transversal a todo el museo -por fin, pandemia mediante, eso se ha puesto muy en evidencia-, el cambio puede muy bien significar redefinir prioridades, reestructurar el organigrama, reasignar roles, establecer nuevos objetivos, construir equipos multidisciplinares, redistribuir recursos. Estamos transitando de la organización jerárquica vertical a una organización más horizontal, más abierta, donde se deshacen los silos, donde la cooperación interna es fundamental.

Necesitamos repensar en clave digital procesos de trabajo, canales de distribución y servicios a los visitantes y usuarios. 


\section{La transformación digital}

La transformación digital de las organizaciones pasa, también, por repensar la orientación de los departamentos de recursos -humanos, económicos, tecnológicos-, que deberán evolucionar hacia una función más estratégica. Así como me gusta definir al museo como conector, a los departamentos de recursos los veo también como conectores de personas, como impulsores de la inteligencia colectiva, que saben poner en valor el capital social interno.

En el contexto actual de incertidumbre y de complejidad, resulta más útil que nunca pararse a reflexionar y plantearse muchas preguntas en clave organizacional:

$\hookrightarrow \quad$ ¿cómo conseguiremos un alineamiento de todo el museo en torno a la visión digital?

$\hookrightarrow \quad$ ¿qué haremos para neutralizar la incertidumbre o el miedo al cambio?

$\hookrightarrow \quad$ ¿cómo podemos ser más eficientes en los procesos mediante la tecnología digital?

$\hookrightarrow$ ¿qué reducción de costes -económicos, de medio ambiente- podríamos conseguir?

$\hookrightarrow \quad$ ¿qué capacidades digitales necesitamos desarrollar internamente? ¿cuáles contratar externamente?

$\hookrightarrow \quad$ el panorama digital, ¿comporta ajustar políticas internas? Por ejemplo, de protección de datos de los usuarios, sobre apertura de datos de las colecciones.

Se hace necesario distribuir la acción digital a través de todo el museo. Tres razones básicas así lo fundamentan. Primero, porque no es sostenible que un solo departamento -ya no digamos una sola persona- asuma y produzca todo el contenido digital. En segundo lugar, porque como lo digital impregna todo el museo, todos deberían estar implicados en su ecosistema digital. Y finalmente también porque con un modelo distribuido proporcionaremos un enriquecimiento de contenidos y de puntos de vista. Vimos durante la pandemia cómo lo digital pasó a ser trabajo de todos en el museo. Hay que aprovechar esta concienciación para solidificar y crecer. Una buena medida para trabajar lo digital desde la transversalidad e implicar al equipo puede ser crear un Grupo de trabajo Digital con integrantes de diferentes departamentos.

Como dicen los expertos de Altimeter, "desarrollar una cultura interna de agilidad digital es tan importante como cualquier iniciativa de estrategia digital". En la estrategia digital del Royal BC Museum (British Columbia) lo puntualizan de manera clara: "Digital platforms and content are the responsibility of single departments. While coordination is necessary to ensure limited resources are working towards common goals, digital creation and idea generation should happen throughout the organization' "ii. El objetivo final no es tener un departamento digital sino, como dijo Loic Tallon cuando era Chief Digital Officer del Met, tener una institución que usa lo digital de manera efectiva para lograr su misión ${ }^{\text {iii }}$. 
Necesitamos organizaciones inteligentes, abiertas a la innovación, con estructuras más flexibles, donde todo esté interconectado, donde las ideas fluyan y se generen nuevas, donde se experimente, donde se arriesgue, donde pensar y tener iniciativa sea reconocido, que se favorezca el aprendizaje continuo.

He expuesto en otros espacios que hay dos elementos fundamentales que cultivar internamente: la actitud -debe ser abierta- y la aptitud. Vamos a profundizar aquí en la aptitud. En general, se necesita en los museos más y mejor formación en competencias digitales, en trabajo colaborativo y en red, en comunicación online, en creación de contenido digital, en movilidad, en accesibilidad digital, en gestión de conocimiento abierto, en visión estratégica. Es cierto que, quien ha querido, ha podido aprovechar las muchas herramientas que hay para hacer auto formación y estar al día, pero las instituciones, y sobre todo las administraciones públicas, deberían facilitar mucho más esta puesta al día. La oferta informativa es muy insuficiente y los perfiles de los profesionales dentro de los museos no están en general suficientemente preparados ni para ser buenos narradores digitales, ni para manejar con suficiencia las herramientas digitales, ni para hacer analítica digital profundizada. Falta formación en todos estos aspectos. En el entorno actual, la formación en competencias digitales se convierte en un factor crítico esencial.

Diversos estudios se han publicado durante el confinamiento y la post-pandemia sobre el impacto COVID en los museos. Ha sido de todo orden, como sabemos. Es de remarcar la coincidencia de diferentes fuentes en la relevancia que adquiere en este nuevo contexto la necesidad de formación y de repensar o elaborar una estrategia digital. Así consta en los informes mostrados abajo, uno del ICOM (Consejo Internacional de Museos) y el otro de NEMO (Network of European Museum Organisations): 


\section{¿Cuáles de los siguientes cambios} está considerando su museo después del cierre?

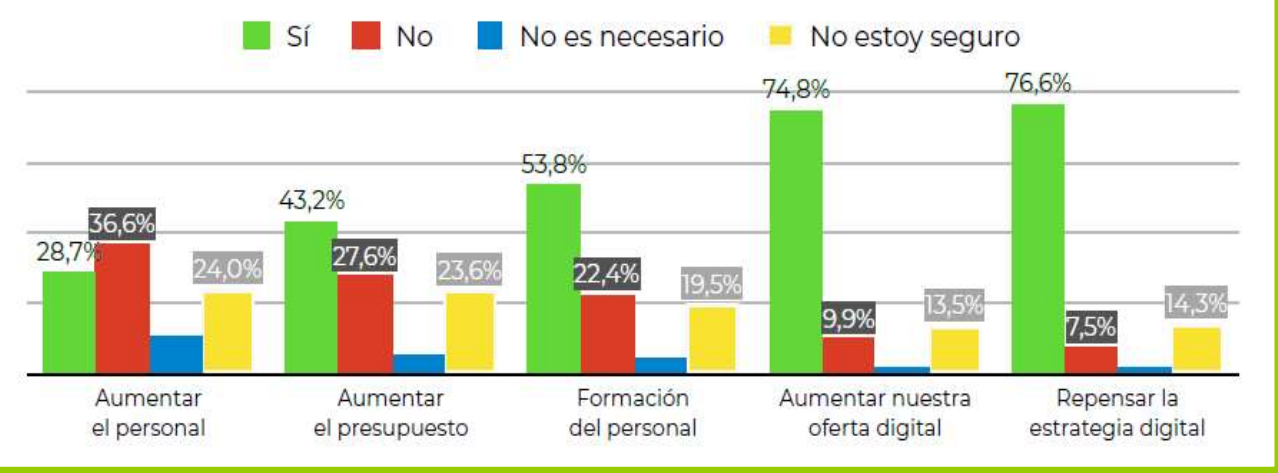

Fuente: Nemo.org

Fig. 2 ICOM (2021). Informe de seguimiento ICOM: Museos, profesionales de los museos y COVID-19, pág. 18.

f yes, what is the priority for your museum?

552 responses

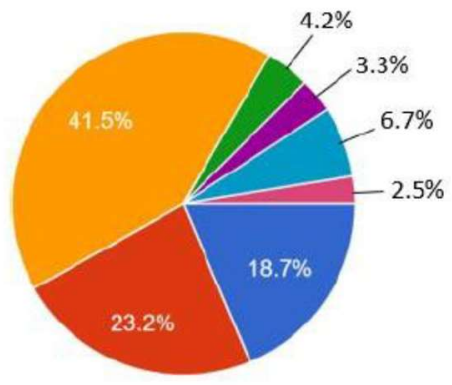

Training staff in digital matters

New digital infrastructure

Building a digital strategy for the museum

Digital visitor research

Defining and tracking metrics of digital success

Not applicable

Other

Fuente: Nemo.org

Fig.3 1NEMO - Network of European Museum Organisations (2021). Follow-up survey on the impact of the COVID-19 pandemic on museums in Europe • pág. 18

Web, colección online, archivos digitales, redes sociales, museografía interactiva, aplicaciones para móviles, 3D, realidad aumentada y muchas otras vías y soportes digitales forman ya parte de las tareas habituales de gestión y de comunicación en los museos. No hay 
museo hoy que se pueda permitir no tener una presencia digital y, como más activa, mejor. Ahora bien, ¿cómo ordenar todo ello? Podemos optar por ir desplegando iniciativas digitales dispersas -aquí una app, allíá un boletín electrónico- que pueden ser muy válidas pero que al final no acaban de cuajar en la construcción de una actuación sólida, o bien podemos afrontar el reto digital de una manera planificada y, sobre todo, alineada con la misión y los objetivos globales del museo. Es decir de una manera estratégica.

Si pasamos nuestros museos por el filtro de la madurez digital que propone la Knight Foundation, veremos que en cuanto a estrategia, la mayoría se mueven entre los estadios de por explorar o emergente. Este modelo marca los hitos a conseguir: disponer de una estrategia ampliamente compartida con todo el museo, evaluar regularmente en función de los objetivos e indicadores trabajados con datos integrados y contar con una colaboración transversal en la planificación de proyectos digitales:

\begin{tabular}{|c|c|c|c|c|}
\hline & & Untapped & Emergent & Realized \\
\hline \multirow{3}{*}{ 总 } & $\begin{array}{l}\text { Strategy creation } \\
\text { and dissemination }\end{array}$ & $\begin{array}{l}\text { - No digital strategy yet, may be } \\
\text { considering one }\end{array}$ & $\begin{array}{l}\text { - Digital strategy may be } \\
\text { developed but not broadly } \\
\text { shared }\end{array}$ & $\begin{array}{l}\text { Digital strategy shared } \\
\text { broadly across museum, may } \\
\text { be included within overall } \\
\text { strategic plan }\end{array}$ \\
\hline & $\begin{array}{l}\text { Goals } \\
\text { development } \\
\text { and outcomes } \\
\text { tracking }\end{array}$ & $\begin{array}{l}\text { Do not have defined goals } \\
\text { or KPIs (Key Performance } \\
\text { Indicators) and/or do not } \\
\text { measure the outcomes }\end{array}$ & $\begin{array}{l}\text { - Some defined goals or } \\
\text { KPIs and some measured } \\
\text { outcomes }\end{array}$ & $\begin{array}{l}\text { - Regularly track against goals } \\
\text { and KPIs with real-time } \\
\text { integrated data }\end{array}$ \\
\hline & $\begin{array}{l}\text { Cross- } \\
\text { institutional } \\
\text { planning of digital } \\
\text { projects }\end{array}$ & $\begin{array}{l}\text { - Little to no planning for } \\
\text { digital, primarily reactive }\end{array}$ & $\begin{array}{l}\text { - Planning by individuals or } \\
\text { individual departments and } \\
\text { centered on specific projects }\end{array}$ & $\begin{array}{l}\text { - Multiple groups or individuals } \\
\text { feed into the planning and } \\
\text { prioritization of digital }\end{array}$ \\
\hline
\end{tabular}

Fuente: Knight Foundation

Fig.4 Digital Readiness and Innovation in Museums Report, pág. 5 Knight Foundation (2020)

Para ello será necesario contar con unas capacidades digitales internas y, probablemente incorporar nuevos perfiles profesionales. Podríamos distinguir entre tres grandes bloques de habilidades necesarias: uno de habilidades digitales generales, que debería tener prácticamente todo el personal del museo, otro de habilidades digitales específicas, en función del puesto de trabajo, y uno de habilidades transversales o "soft" skills, tales como saber planificar proyectos, resolver problemas, trabajar en equipo, liderar, comunicar, ser flexibles al cambio, tener empatía y muchas más: 


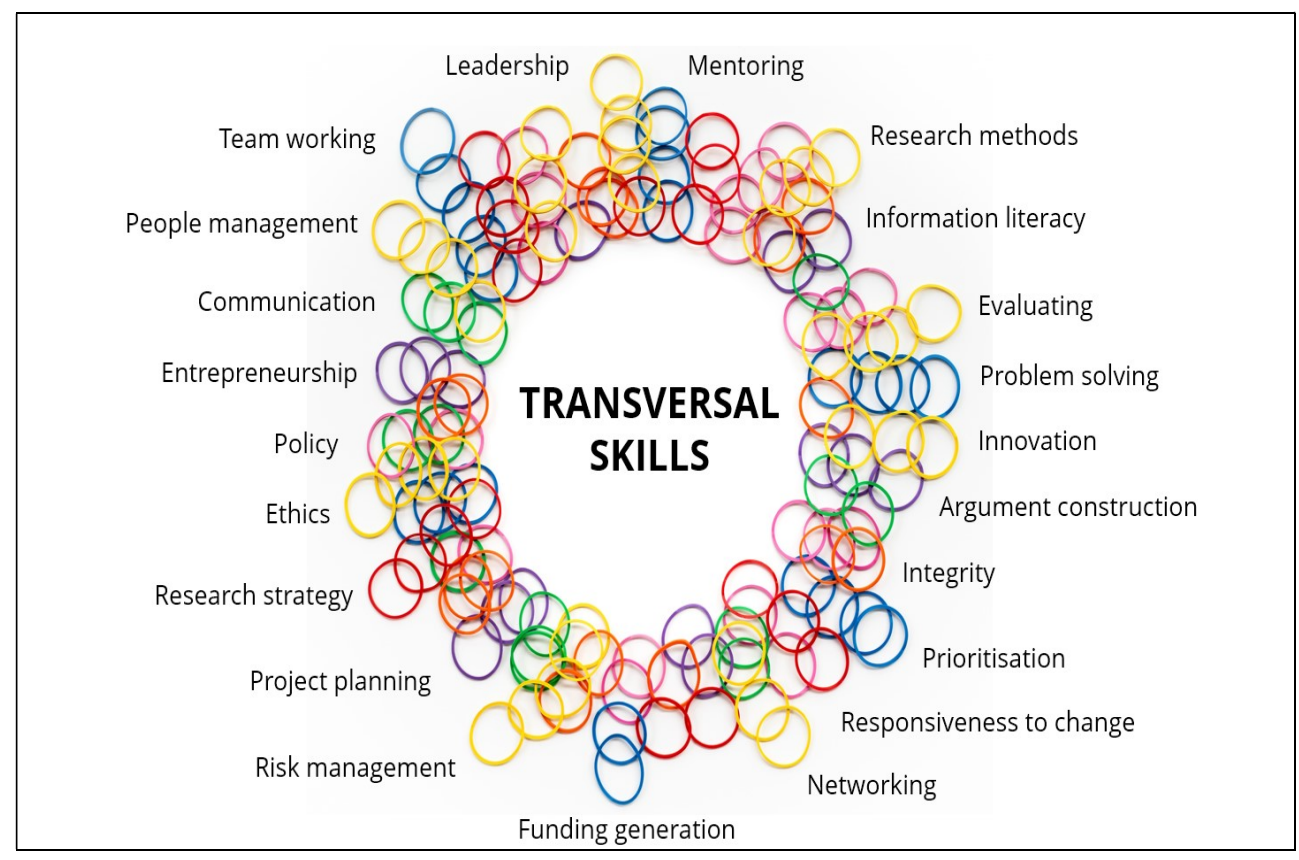

Fuente: agaur.gencat.cat

Fig.5 Euraxess Catalonia. "Herramientas para el desarrollo profesional”

En cuanto a las capacidades digitales específicas, para ayudar a visibilizar mejor la necesidad de formarse en habilidades digitales en museos y mostrar qué requisitos se pedían en ofertas reales de trabajo en museos de todo el mundo, hace un tiempo empecé a recopilar ejemplos para mis estudiantes de máster y luego pensando en una posible utilidad para museos y universidades, expandí el informe con más ejemplos, datos, gráficos, extractos de informes y artículos tanto de museos como de consultoras internacionales en estrategia. Compartiré ahora algunos de los gráficos y reflexiones de este informe, Digital Skills and Profiles in Museums today, que se puede descargar libremente ${ }^{\text {iv }}$. Hay que reconocer que ha habido mucho esfuerzo individual por parte de muchos profesionales de museos para auto-formarse y que ha habido iniciativas parciales desde algunas administraciones como la Generalitat de Catalunya o el Eusko Jaurlaritza-Gobierno Vasco dirigidas a proporcionar formación a personal de sus museos. Pero hay que abrir mucho más el abanico, tanto de oferta formativa como de destinatarios.

En primer lugar para poder avanzar es preciso que haya un liderazgo digital. Ello no quiere, claro está, decir que los directivos tengan que ser expertos en tecnología sino que deben tener la visión de lo esencial de lo digital hoy, su potencial para el museo y facilitar el desarrollo de iniciativas digitales, que fortalezcan la misión y los objetivos del museo, así como articular un entorno de aprendizaje continuo. Muy categórica, Jane Finnis calibra la laguna existente, especialmente en posiciones de liderazgo, respecto a la comprensión de la dimensión digital: "It is around the understanding of digital where we have found significant gaps, particularly 
in leadership positions [...] Our [museums and heritage] sector needs leaders that can be informed, reflective, responsive and active in their digital understanding, so that as organisations we can build a more digitally fluent workforce and create digitally mature relationships with our audiences"v.

Hablamos de digitalización, pero al final, como dice Risto Sarvas, se trata de capacidades, habilidades y de maneras de trabajar, lo que significa repensar la estructura organizacional y el liderazgo ${ }^{\text {vi }}$. Sucede a menudo, sin embargo, que los responsables no son conscientes de las necesidades de desarrollo de las capacidades digitales del equipo. Así lo constataron los Museos Nacionales de Escocia cuando emprendieron un proceso interno de desarrollo digital que denominaron Developing Digital Potential. En palabras de su director de Digital Media, Rob Cawston, "The initial conversations with team leaders and managers [at National Museums Scotland] revealed that they are often not aware of the digital skills development needs of their staff [...] The Developing Digital Potential process was seen as a valuable way to address these issues and to consider individual development needs"vii.

El equipo británico de Culture24 establece tres elementos de las habilidades digitales: competency (acción), o la habilidad para usar una herramienta digital; capability (intención), o cómo esa habilidad se aplica con éxito a una tarea; y literacy (reflexión), ser capaz de evaluar lo apropiado de esas competencias y habilidades. Estos tres elementos juntos son esenciales para construir la robustez digital ${ }^{\text {viii. }}$.

Queda mucho camino por recorrer. Lo sabemos y lo corroboran los datos del estudio ya mencionado de NEMO: el $45 \%$ de los museos encuestados manifestaron una falta de competencias digitales:

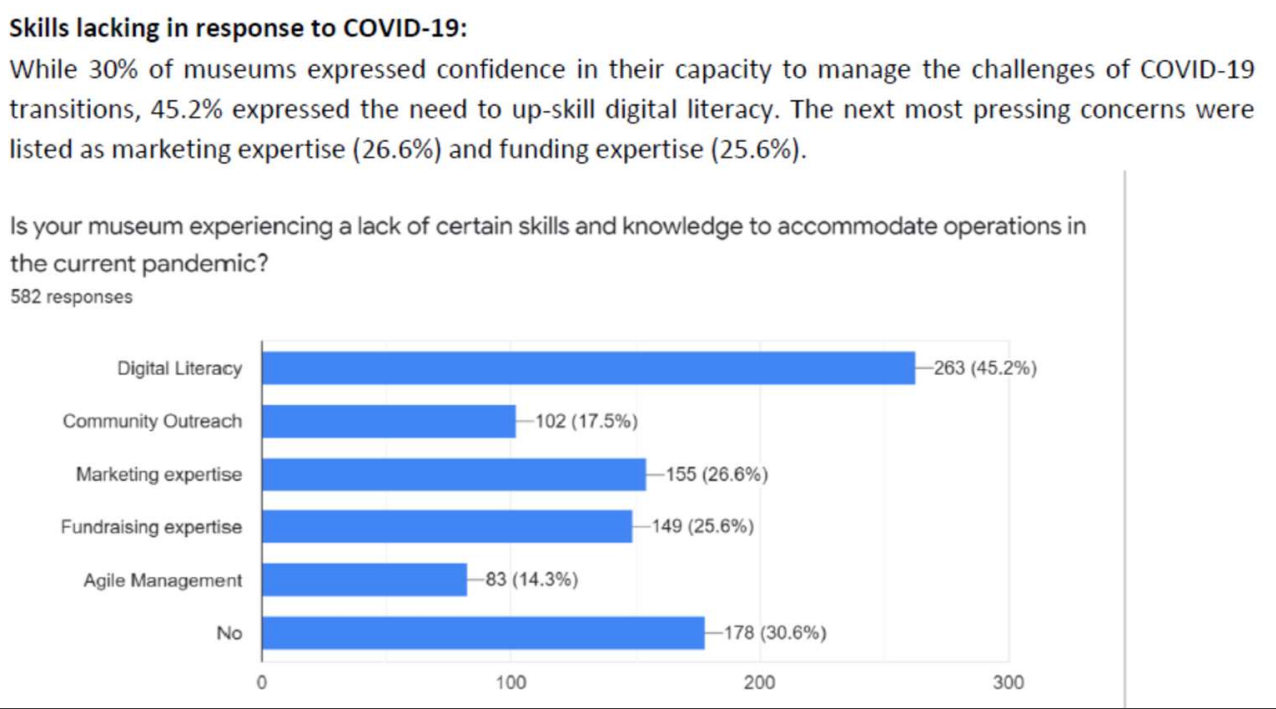

Fuente: Nemo.org

Fig.6 NEMO - Network of European Museum Organisations (2021). Follow-up survey on the impact of the COVID-19 pandemic on museums in Europe . Final Report, pág. 24 
Parece pues evidente que en este entorno post-pandémico una de las prioridades debe ser la mejora de las competencias digitales de los profesionales de museos. Absolutamente imprescindible si, además, tenemos en cuenta que la contratación de nuevos perfiles profesionales en estos tiempos de estrechez presupuestaria va a ser muy difícil, sobre todo para los museos pequeños y medianos.

Existen notables iniciativas para el reskilling y upskilling en el sector de museos. Por citar algunos, los MOOCS (cursos masivos abiertos online) de Mu.SA - MUseum Sector Alliance (https://mooc.cti.gr/musa.html) $\quad$ y $\quad$ de $\quad$ Europeana (https://www.europeanschoolnetacademy.eu/courses/course-

v1:Europeana+Culture_EN+2021/about), o la formación de $\underline{\text { FutureLearn }}$ (https://www.futurelearn.com/courses/collections/digital-skills-workplace) y de Culture 24 (https://digipathways.co.uk/resources/). En el ámbito hispanohablante ofrecen formación

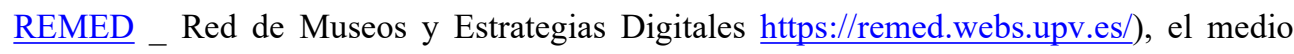
Postgrado de Estrategia Digital para Organizaciones Culturales (https://estudios.uoc.edu/es/masters-posgrados-especializaciones/especializacion/arteshumanidades/estrategia-digital-organizaciones-culturales-mnac/presentacion),

coorganizado por la UOC (Universitat Oberta de Catalunya) y el Museu Nacional d'Art de Catalunya, o iniciativas en Latinoamérica como Open Museum (https://indesvirtual.iadb.org/course/view.php?id=1887), y la Fundación TyPA - Teoría y Práctica de las Artes, (https://www.typa.org.ar/es/museos.php). Pero se encuentra a faltar una oferta formativa digital integral en el ámbito de patrimonio desde los organismos públicos en nuestro país. Un sólido programa público de capacitación digital que cubriera las habilidades digitales generales de cualquier profesional hoy y las necesidades específicas en función del trabajo a realizar en digital en el museo y que abarcara diferentes niveles formativos sería un objetivo deseable - ¿exigible? Y urgente. Comparto plenamente la afirmación de MuSA: "Increasing digital literacy and confidence among museum professionals is a crucial aspect of supporting the digital transformation of museums and enabling change" ${ }^{\text {,ix }}$.

Efectivamente, es crucial, solo con una buena capacitación digital podrán los museos hacer la transición digital en el contexto post-pandémico y despuntar en nuevas vías de conectar con los visitantes y usuarios, conseguir el preciado digital engagement, ofrecerles una óptima mediación digital y, en definitiva, prestarles un mejor servicio dónde, cuándo y cómo lo prefieran. A la vez que convertir los museos en organizaciones ágiles donde las herramientas digitales refuercen el trabajo colaborativo. Sin olvidar el talento senior, como a menudo nos recuerda Laura Rosillox.

Sin olvidar tampoco, que la tecnología digital es solo una herramienta no el fin, que lo presencial tiene un valor singular insustituible y que los cambios se están produciendo desde múltiples flancos. Decía hace poco para el Anuario Digital de Cultura que "junto a la propulsión de la esfera digital, de la mano de la pandemia, han llegado más cambios a los museos, otros impulsos como son la mayor focalización en la audiencia, el protagonismo de 
la colección, el giro a los públicos de proximidad, la intensificación de la participación de los usuarios, características todas ellas esenciales a la propia misión de los museos y que el nuevo escenario ha proyectado a primera línea. Aunando acción digital y analógica el museo saldrá reforzado. Hibridar programación, contenidos, formatos o servicios potenciará el impacto y la relevancia social de nuestros museos"

Al final, resulta inseparable la capacitación digital del propósito social. Dice Ross Parry: "Digital literacy and social purposefulness are linked. In fact, they're inter-dependent. We can't think about digital transformation in arts and heritage organisations without considering the socially purposeful aims of those organisations. Similarly, we can't think about being a socially purposeful organisation today without reflecting on the institution's digital capabilities, the digital dimensions to modern society, and the digital contexts of audiences' lives"xii. La sociedad es digital, los museos para rendir un buen servicio y ganar relevancia social necesitan mejorar sus competencias digitales.

\section{Algunas conclusiones}

$\rightarrow$ Existe una tremenda necesidad de una mejor formación y perfeccionamiento en las capacidades digitales para que los museos puedan abordar con éxito los desafíos y la transformación digital en el contexto actual.

$\rightarrow$ Cualquier rol debe estar preparado para lidiar con lo digital con competencia, por lo que el aprendizaje a lo largo de la vida será una constante.

$\rightarrow$ Los museos necesitan incorporar nuevos perfiles digitales altamente capacitados en sus equipos.

$\rightarrow$ Los museos en general se quedaron atrás en cuanto a transformación digital. El segundo mejor momento para abordarla es ahora. Para ello,

$\rightarrow$ Lo digital debe abordarse de manera integral y estratégica en toda la organización.

Los ingredientes de la fórmula digital serán específicos de cada museo, de su misión, objetivos, colecciones, públicos. Pero hay algunos básicos que, a mi entender, no deberían faltar nunca: la orientación al público, la contextualización de las colecciones, la interpretación digital en salas y online, una mayor difusión de la labor investigadora y sus resultados, el trabajo colaborativo y, óptimamente, el acceso abierto a los datos, imágenes, contenidos para su uso y reutilización libre. Esa apertura conduciría a los museos directo a su misión de extender el conocimiento virtualmente a todo el mundo. 


\footnotetext{
' Altimeter (2015). Crafting a Digital Strategy: a Process \& Checklist for Digital Strategy Development, pág 19. http://www2.prophet.com/crafting-a-digital-strategy

ii Royal BC Museum Digital Strategy 2015-2020 (2016), pág 5.

https://royalbcmuseum.bc.ca/documents/105659/digital-strategy

iiiTallon, Loic (2017). "The end goal is not to have a digital department, buit for an institution to use digital effectivelty to achieve its mission" a "Digital is more than a Department, it is a Collective Responsibility", https://www.metmuseum.org/blogs/now-at-the-met/2017/digital-future-at-the$\underline{\text { met }}$

iv Rodà, Conxa (2020). Digital Skills and Profiles in Museums today.

Versión breve, $46 \mathrm{pp}$, descargable en https://www.linkedin.com/posts/conxaroda digital-profilesskills-in-museums-today-activity-6699727572890189824-7HJ7
}

Versión completa, 98 pp, descargable en DOI: 10.13140/RG.2.2.13796.40321

" Finnis, Jane (2020). "Why digitally literate leadership is so important right now".

https://medium.com/@janefinnis/why-digitally-literate-leadership-is-so-important-right-now3c2d9b214dde

vi Sarvas, Risto (2020), entrevistado por Kajsa Hartig https://www.idek.se/meetup-3-risto-sarvasprofessor-of-practice-aalto-university

vii Cawston, Rob (2020). "Sustainable processes for building digital skills", https://digipathways.co.uk/sustainable-processes-building-digital-skills/. Ver también Karin de Wild "Developing staff digital skills: approach \& process", https://digipathways.co.uk/developing-staffdigital-skills-approach-process/

viii Culture 24 (2020). The digital transformation agenda and GLAMs - Culture24 findings and outcomes, pág. 9.

https://pro.europeana.eu/files/Europeana Professional/Publications/Digital\%20transformation\%20rep orts/The\%20digital\%20transformation\%20agenda\%20and\%20GLAMs $\% 20$ -

\%20Culture24,\%20findings\%20and\%20outcomes.pdf

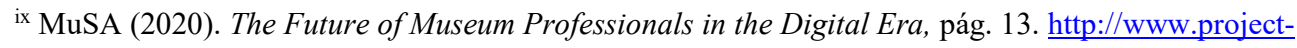
musa.eu/wp-content/uploads/2021/03/Mu.SA-e-book.pdf

× Ver entrevista a Laura Rosillo en DigitalFone (julio de 2020). https://digital-fone.com/talentosenior-laura-entrevista-laura-rosillo/

${ }^{x i}$ Rodà, Conxa (2021). "La ansiada redimensión de lo digital: una mirada internacional al impacto del COVID-19 en los museos" en Anuario AC/E de cultura digital 2021, págs. 59-60. https://www.accioncultural.es/es/anuario2021

xii Parry, Ross (2019). "Socially purposeful digital skills". https://one-by-one.uk/2019/03/11/sociallypurposeful-digital-skills/ 


\section{Bibliografía recomendada}

Europeana (2020). Digital transformation in the time of COVID-19. $59 \mathrm{pp}$.

https://pro.europeana.eu/post/digital-transformation-in-the-time-of-covid-19-sensemaking-workshops-findings-and-outcomes

Eve Museografía (2019). "Despliegue de Habilidades Digitales en los Museos". https://evemuseografia.com/2019/12/09/despliegue-de-habilidades-digitales-en-losmuseos/

ICOM Webinars. "Capacitación para profesionales de los museos". https://icom.museum/es/news/webinars-capacitacion-museos/

Marty, Paul (2021). "Contributions of technology museum professionals in times of crisis", ponencia en el congreso CIMED 2021. https://www.youtube.com/watch?v=EJsmIQpBohl Observatori de competències digitals i ocupabilitat (2021). Informe: Indicadores de Competencias Digitales $y$ Ocupabilidad. 70 pp. Descargable en https://imancorpfoundation.org/i-observatorio-sobre-digitalizacion-y-empleo/

One by One (2019). Understanding the digital skills \& literacies of UK museum people. Phase Two Report

https://one-by-one.uk/2019/05/21/phase-2-findings/ DOI:

https://doi.org/10.29311/2018.02

One by One (2018). Mapping the Museum Digital Skills ecosystem. https://one-byone.uk/2018/03/23/phase-1-findings/

Skills Platform and Amar, Zoe (2020). Charity Digital Skills Report 2020. http://report.skillsplatform.org/charity-digital-report-2020/ 